\title{
MECANISMOS QUÍMICOS E MINERALÓGICOS DE TRANSFORMAÇÃO DA MAGNESIOFERRITA DE SOLO DERIVADO DE TUFITO, DA REGIÃO DO ALTO PARANAÍBA, MG
}

\author{
Fernando Dias da Silva* e Antonio Taranto Goulart \\ Departamento de Química, Centro Universitário de Patos de Minas, CP 485, 38702-054 Patos de Minas - MG, Brasil \\ Paulo Rogério da Costa Couceiro \\ Departamento de Química, Universidade Federal do Amazonas, 69077-000 Manaus - AM, Brasil \\ José Domingos Fabris \\ Departamento de Química, Instituto de Ciências Exatas, Universidade Federal de Minas Gerais, 31270-901 Belo Horizonte - MG, \\ Brasil
}

Recebido em 13/11/08; aceito em 3/4/09; publicado na web em 26/8/09

\begin{abstract}
CHEMICAL AND MINERALOGICAL MECHANISMS RELATED TO THE TRANSFORMATION OF MAGNESIOFERRITE, IN A SOIL FORMING ON TUFFITE IN ALTO PARANAÍBA, MG. Magnetic soils forming on tuffite of the region of Alto Paranaíba, Minas Gerais, Brazil, usually contain iron-rich spinels exceptionally rich in magnesium and titanium. In this work, samples of the magnetically separated portion from the sand fraction of a Brunizém (Chernossolo) and from its mother-rock material were analyzed with synchrotron X-ray diffraction and ${ }^{57} \mathrm{Fe}-\mathrm{Mössbauer}$ spectroscopy. Magnesioferite $\left(\mathrm{MgFe}_{2} \mathrm{O}_{4}\right)$ and maghemite (its pure nonstoichiometric spinel structure, $\mathrm{Fe}_{8 / 3} \oplus_{1 / 3} \mathrm{O}_{4}$, where $\oplus$ = cation vacancy, corresponds to $\gamma \mathrm{Fe}_{2} \mathrm{O}_{3}$ ) were the magnetic iron oxides so identified. Basing on these data, a consistent chemical-mineralogical model is proposed for the main transformation steps involving these iron oxides in the pedosystem, starting on magnesioferrite to finally render hematite $\left(\alpha \mathrm{Fe}_{2} \mathrm{O}_{3}\right)$, passing through maghemite as an intermediate specie.
\end{abstract}

Keywords: synchrotron light; maghemite, Mössbauer; iron oxide.

\section{INTRODUÇÃO}

Tufitos são rochas resultantes da deposição, compactação e cimentação de materiais piroclásticos (cinzas e poeiras vulcânicas). A origem vulcânica confere textura, estrutura e demais aspectos comparáveis a outras espécies de depósitos sedimentares clásticomagmáticos, classificados como rochas ígneas extrusivas. ${ }^{1}$ São materiais friáveis e porosos, que tendem a produzir espessos mantos de material intemperisado. ${ }^{2}$

Os tufos vulcânicos distribuídos na região do Alto Paranaíba formam a litologia de tufito, com expressiva variabilidade, tanto vertical quanto horizontalmente, em termos de composição química e mineralógica. ${ }^{3}$ Os solos derivados de tufito da região contêm espinélios de ferro excepcionalmente ricos em magnésio e titânio. ${ }^{4,5}$ As frações grosseiras do solo derivado tendem a preservar proporções variáveis de minerais magnéticos, especificamente magnetita e maghemita. ${ }^{6-9}$ Recentemente, foi ineditamente identificada e descrita a magnesioferrita $\left(\mathrm{MgFe}_{2} \mathrm{O}_{4}\right)$ desse litodomínio, com magnetização de saturação, $\sigma=21 \mathrm{~J} \mathrm{~T}^{-1} \mathrm{~kg}^{-1}$. 10-12

$\mathrm{O}$ presente trabalho foi dedicado à caracterização, na busca de um modelo plausível de transformações envolvendo óxidos de ferro isoestruturais ao espinélio (maghemita e magnesioferrita), de materiais separados de uma amostra de solo (horizonte C) e de seu material de origem (tufito consolidado), coletados na região do Alto Paranaíba, MG.

\section{PARTE EXPERIMENTAL}

Foram coletadas duas amostras, uma de solo, classificado como Brunizém (Chernossolo - horizonte CR), de acordo com o Sistema Brasileiro de Classificação de Solos, ${ }^{13}$ e outra de tufito consolidado

\footnotetext{
*e-mail: fernando.silva@unipam.edu.br
}

(rocha), no município de Patos de Minas (coordenadas geográficas do sítio de amostragem, $18^{\circ} 39^{\prime} 32,9^{\prime \prime} \mathrm{S}$ e 46 16' 53,5” O), região do Alto Paranaíba, no estado de Minas Gerais.

A amostra de solo foi desagregada manualmente, seca ao ar e passada em peneira com malhas de $2 \mathrm{~mm}$ de abertura, obtendo-se a fração terra fina seca ao ar (TFSA). Da TFSA, foi obtida a fração areia (diâmetro médio, $\phi_{m}=0,05$ a $2 \mathrm{~mm}$ ), por tamisação em peneira com malha de $0,05 \mathrm{~mm}$ e lavagens sucessivas com água destilada. A amostra de tufito (material consolidado/ rocha) foi triturada em gral de aço inoxidável não-magnético. Porções de grãos magnéticos das amostras de areia do solo e de material consolidado triturado foram magneticamente separadas, com um ímã de mão, e lavadas por várias vezes, com água. Os materiais assim obtidos, rotulados AP (concentrado magnético da fração areia do solo) e TC (concentrado magnético do tufito consolidado/rocha), foram efetivamente estudados neste trabalho. As amostras AP e TC foram quimicamente tratadas com ditionito-citrato-bicarbonato de sódio (DCB),${ }^{14}$ visando a eliminar ou a reduzir o teor de hematita presente. Para as análises químicas, realizadas em triplicata, procedeu-se à dissolução mediante fusão alcalina, utilizando-se uma mistura equimolar de $\mathrm{Na}_{2} \mathrm{CO}_{3}+\mathrm{K}_{2} \mathrm{CO}_{3}$ em cadinhos de platina, de cerca de $0,1 \mathrm{~g}$ (precisão analítica) da amostra, a $1000^{\circ} \mathrm{C}$, por $1 \mathrm{~h}$, seguida da solubilização do resíduo com a adição de $\mathrm{HCl}\left(6 \mathrm{~mol} \mathrm{~L}^{-1}\right)$ e filtração. O filtrado foi volumetricamente aferido para $250,0 \mathrm{~mL}$. $\mathrm{O}$ teor de Fe total foi quantificado por dicromatometria (titulação com $\mathrm{K}_{2} \mathrm{Cr}_{2} \mathrm{O}_{7}$ a $0,02 \mathrm{~mol} \mathrm{~L}^{-1}$ ), após tratamento da solução final com $\mathrm{NH}_{3 \text { (aq), }}$, para se eliminar a interferência da platina. ${ }^{15} \mathrm{O}$ teor de $\mathrm{SiO}_{2}$ foi determinado gravimetricamente, por fluorização do resíduo da fusão, em cadinho de platina, sob aquecimento em banho de areia, e posterior calcinação em mufla, a $1000{ }^{\circ} \mathrm{C} .{ }^{16} \mathrm{~A}$ quantificação elementar de Al, Ti, Ca, Mg, Mn e Ni foi feita em espectrômetro de absorção atômica marca Hitachi Z-8200, equipado com um corretor de fundo, por efeito Zeeman polarizado, e a de $\mathrm{Na}$ e K, em fotômetro 
Tabela 1. Composição química total das amostras TC e AP

\begin{tabular}{|c|c|c|c|c|c|c|c|c|}
\hline Amostra & $\mathrm{Fe}_{2} \mathrm{O}_{3}$ & $\mathrm{MnO}$ & $\mathrm{NiO}$ & $\mathrm{Cr}_{2} \mathrm{O}_{3}$ & $\mathrm{TiO}_{2}$ & $\mathrm{Al}_{2} \mathrm{O}_{3}$ & $\mathrm{MgO}$ & TOTAL \\
\hline \multicolumn{9}{|c|}{ 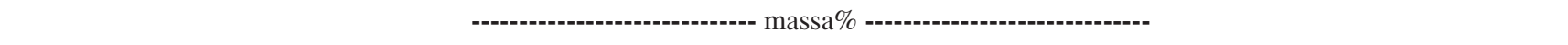 } \\
\hline $\mathrm{TC}$ & $74,6(1)$ & $1,6(3)$ & $1,5(1)$ & $0,25(1)$ & $7,4(3)$ & $1,2(1)$ & $11,7(2)$ & $98,25(2)$ \\
\hline AP & $75,50(2)$ & $0,90(1)$ & $0,91(3)$ & $1,34(5)$ & $10,03(2)$ & $1,61(6)$ & $3,95(5)$ & $94,24(3)$ \\
\hline
\end{tabular}

Os números entre parênteses são incertezas do valor numérico, estimadas pelo desvio padrão de resultados de procedimentos analíticos, desde a abertura, em triplicata, e expressas sobre o último algarismo significativo, do valor numérico da quantidade física correspondente.

de chama modelo CELM FC-180. As medidas de magnetização de saturação $(\sigma)$ foram realizadas em um magnetômetro portátil, a campo magnético fixo de 0,3 tesla. ${ }^{17}$ Os difratogramas de raios $\mathrm{X}$ foram coletados no Laboratório Nacional de Luz Síncrotron (LNLS, Campinas, SP). Os difratogramas foram numericamente ajustados, com base no refinamento estrutural pelo algoritmo Rietveld, ${ }^{18}$ utilizando-se o programa FullProf, ${ }^{19}$ com a função pseudo-Voigt. Adotou-se a estrutura cristalográfica cúbica, grupo espacial $F d 3 m$, para os óxidos de ferro com estrutura do espinélio. Foram refinados os parâmetros de célula unitária, térmico isotrópico, escala, linha de base (função polinomial de 4 coeficientes), largura de linha a meia altura, $\mathrm{FWHM}^{2}=\mathrm{Utg}^{2} \theta+\mathrm{V} \operatorname{tg} \theta+\mathrm{W}$ (fórmula de Caglioti), ${ }^{20}$ forma e assimetria das reflexões, origem zero para escala de varredura $2 \theta$ e coordenada espacial do ânion, na estrutura cristalina.

Os espectros Mössbauer foram coletados a $298 \mathrm{~K}$ (temperatura do ambiente) e a $110 \mathrm{~K}$ (criostato de banho de nitrogênio líquido), em montagem convencional de transmissão, com uma fonte de ${ }^{57} \mathrm{Co} /$ $\mathrm{Rh}, \mathrm{de} \sim 30 \mathrm{mCi}$. A massa da amostra para a medida foi estimada para conter $\sim 10 \mathrm{mg} \mathrm{Fe} \mathrm{cm}^{-2}$. Os deslocamentos isoméricos foram corrigidos em relação ao $\alpha \mathrm{Fe}$, padrão também usado para a calibração da escala de velocidade Doppler. Os dados foram ajustados numericamente com funções lorentzianas, pelo método dos mínimos quadrados, utilizando-se o programa de computador NORMOS ${ }^{\mathrm{TM}}-90 .{ }^{21}$

\section{RESULTADOS E DISCUSSÃO}

A composição química das amostras AP e TC são apresentadas na Tabela 1. Os altos teores de ferro, expressos na base de óxido, da ordem de 74,6 massa\% e 75,5 massa $\% \mathrm{Fe}_{2} \mathrm{O}_{3}$, respectivamente, para as amostras TC (rocha) e AP (solo), estão condizentes com a natureza das amostras estudadas, que são constituídas, essencialmente, por óxidos de ferro.

Na Tabela 2, são apresentados os valores de magnetização de saturação para os concentrados magnéticos das amostras tratadas com $\mathrm{DCB}^{14} \mathrm{e}$ das sem tratamento químico. Observa-se aumento significativo da magnetização, pelo tratamento com $\mathrm{DCB}$, em relação àquelas sem tratamento, da ordem de 20 a 30\%, para as amostras de TC e AP, respectivamente. Estes resultados não surpreendem, na medida em que o tratamento químico concentra seletivamente óxidos de ferro magnéticos, como maghemita e magnesioferrita.

Tabela 2. Medidas de magnetização de saturação (s $\mathrm{s}_{\mathrm{s}}$ das amostras

\begin{tabular}{lcc}
\hline \multirow{2}{*}{ Tratamento Realizado } & \multicolumn{2}{c}{ Magnetização Relativa s/J T $\mathrm{kg}^{-1}$} \\
& TC & AP \\
\hline ST & 11,6 & 33,6 \\
DCB & 14,0 & 44,7 \\
\hline
\end{tabular}

ST - amostra sem tratamento químico

O difratograma de raios X (método do pó), obtido para a amostra TC (Figura 1), apresenta picos de difração que permitem identificar a presença de óxidos de ferro com estrutura de espinélio, como a magnesioferrita (Mf) e a maghemita (Mh). ${ }^{10,11}$ Uma vez que os picos relativos a esses minerais, principalmente a menores ângulos, são bastante coincidentes (ambos têm estrutura cúbica), foi feita a decomposição do reflexo de maior intensidade, relativo ao plano (311), usando-se a função Voigt (Figura 1), o que permitiu atribuir reflexões, a ângulos menores, à magnesioferrita ${ }^{22}$ (ficha JCPDS (1980) número 36-0398, dimensão característica da célula unitária cúbica, $\boldsymbol{a}=0,83873(6) \mathrm{nm}$ ) e, a ângulos maiores, à maghemita (ficha JCPDS (1980) número 39-1346, dimensão característica da célula unitária cúbica, $\boldsymbol{a}=0,83512(6) \mathrm{nm}$ ). Os picos de difração de raios $\mathrm{X}$ da amostra AP (Figura 2) também foram comparados com dados referentes à magnesioferrita e à maghemita. $\mathrm{O}$ reflexo que ocorre entre os ângulos $2 \theta, 34,2^{\circ}$ a $34,9^{\circ}$, correspondente ao plano (311), da maghemita e da magnesioferrita, foi decomposto e os resultados (Figura 2) descartam a ocorrência de hematita, no material analisado.

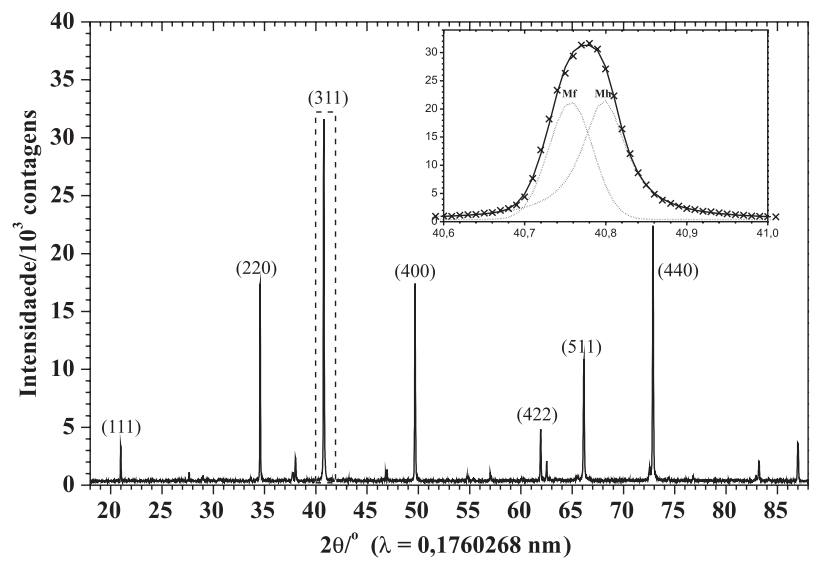

Figura 1. Difratograma de raios X da fração magnética do tufito (TC); em destaque a decomposição do reflexo correspondente ao plano (3 11 1)

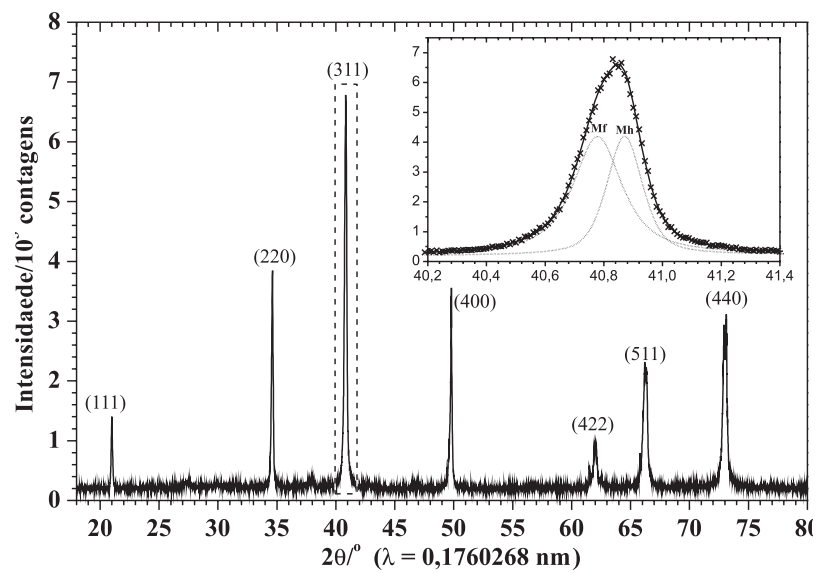

Figura 2. Difratograma de raios $X$ da fração magnética do solo derivado do tufito (AP); em destaque a decomposição do reflexo correspondente ao plano (3 11 1) 
O espectro Mössbauer da amostra TC obtido a 298 K (Figura 3) foi ajustado com um modelo de decomposição em quatro subespectros: três sextetos, devidos a acoplamento hiperfino em estruturas magneticamente ordenadas, e um dupleto, pouco intenso, (super) paramagnético. Os parâmetros correspondentes das fases magnéticas são mostrados na Tabela 3. O subespectro com deslocamento isomérico $\delta=0,34 \mathrm{~mm} \mathrm{~s}^{-1}$ e campo hiperfino $B_{h f}=48,90$ tesla é atribuível à maghemita, ${ }^{23,24}$ enquanto os outros dois, com deslocamentos isoméricos $\delta=0,38$ e $0,37 \mathrm{~mm} \mathrm{~s}^{-1}$ e campos hiperfinos $B_{h f}=44,5$ e 46,6 tesla, são característicos de íon férrico alto spin e correspondem, respectivamente, aos sítios de coordenação octaédrica e tetraédrico da magnesioferrita. ${ }^{10,11,25} \mathrm{~A}$ falta de evidência espectral característica de $\mathrm{Fe}^{2+}$ ou de ferro de valência mista $\left(\mathrm{Fe}^{2,5+}\right)$ exclui a ocorrência de magnetita.

O espectro Mössbauer obtido para a amostra AP a 298 K (Figura 3) mostra sextetos bastante complexos, com padrão espectral de linhas largas e assimétricas. O espectro foi ajustado com três subespectros magnéticos, definidos por linhas de ressonância lorentzianas e, simultaneamente, por distribuição de campo hiperfino (Figura 3). Os parâmetros hiperfinos assim obtidos são apresentados na Tabela 3. Os três subespectros magnéticos para $\mathrm{Fe}^{3+}$ resultaram em $B_{h f}=47,70$ e 49,06 tesla correspondentes, respectivamente, aos sítios de coordenações tetraédrica e octaédrica da magnesioferrita, e outro, com valor médio $B_{h f}=50,47$ correspondente aos sítios de coordenações tetraédrica e octaédrica, da maghemita. ${ }^{10,11,26,27} \mathrm{Um}$ quarto subespectro, mais largo e assimétrico, para o qual a distribuição de campo hiperfino revela um valor médio provável entre $B_{h f}=44,4$ e 47,0 tesla, pode ser resultante da mistura de fases magnesioferrita + maghemita, de pequenos tamanhos de partículas, ${ }^{23}$ com redução de campo resultante do fenômeno de excitação magnética coletiva. ${ }^{28}$ Mesmo para a amostra AP, não foi encontrada evidência espectral da presença de hematita, que seria, presumivelmente, o produto final, na escala de intemperização dos minerais magnéticos encontrados (maghemita e magnesioferrita). Isso pode ser atribuído ao tratamento químico com DCB,${ }^{14}$ a que foram submetidas as amostras. Admitindose, inicialmente, que ambas as espécies magnéticas presentes nas amostras apresentem comportamentos químicos similares, frente ao tratamento, o enriquecimento relativo, quando se vai da rocha para o solo, observado das áreas espectrais Mössbauer, permite sugerir ser a maghemita mais estável do que a magnesioferrita frente à ação da mistura DCB.

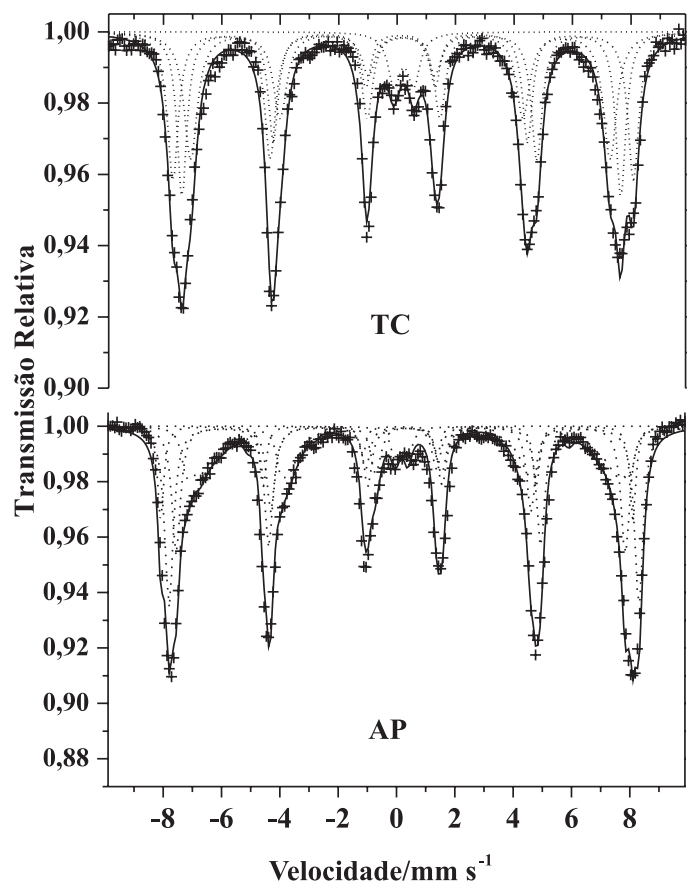

Figura 3. Espectros Mössbauer da fração magnética do tufito (TC) e do seu solo derivado (AP) obtidos a $298 \mathrm{~K}$

As medidas Mössbauer a baixa temperatura dão informações mais detalhadas sobre as estruturas hiperfinas dos óxidos de ferro magnéticos. No presente estudo, a primeira análise, ainda qualitativa, do espectro Mössbauer a $110 \mathrm{~K}$ para a amostra TC (Figura 4) revela o ordenamento magnético de parte da estrutura superparamagnética, observada à temperatura do ambiente, através da diminuição da intensidade do dupleto central. ${ }^{23}$ Para essa temperatura, o ajuste

Tabela 3. Parâmetros Mössbauer dos espectros das amostras TC e AP, obtidos a $298 \mathrm{~K}$

\begin{tabular}{|c|c|c|c|c|c|c|c|}
\hline Amostra & Sítio & $\delta / \mathrm{mm} \mathrm{s}^{-1}$ & $\Delta, \varepsilon / \mathrm{mm} \mathrm{s}^{-1}$ & $B_{h f} / \mathrm{T}$ & $A R / \%$ & $\Gamma / \mathrm{mm} \mathrm{s}^{-1}$ & $\mathrm{DC}$ \\
\hline \multirow[t]{4}{*}{$\mathrm{TC}$} & [Mf] & $0,38(3)$ & $-0,03(1)$ & $44,50(5)$ & $29,0(1)$ & $0,55(2)$ & \\
\hline & $\{\mathrm{Mf}\}$ & $0,37(1)$ & $0,03(1)$ & $46,60(2)$ & $35,2(5)$ & $0,40(1)$ & \\
\hline & $\mathrm{Mh}$ & $0,34(1)$ & $-0,01(1)$ & $48,90(2)$ & $30,3(1)$ & $0,45(2)$ & \\
\hline & $\mathrm{Fe}^{3+}$ & $0,36(2)$ & $0,68(2)$ & & $5,5(2)$ & $0,50(2)$ & \\
\hline \multirow[t]{9}{*}{ AP } & [Mf] & $0,39(1)$ & $-0,01(2)$ & $47,70(2)$ & $16,2(3)$ & & \\
\hline & $\{\mathrm{Mf}\}$ & $0,40(1)$ & $-0,03(3)$ & $49,06(2)$ & $25,6(2)$ & & \\
\hline & $\mathrm{Mh}$ & $0,28(2)$ & $-0,02(1)$ & $50,47(2)$ & $27,5(1)$ & & \\
\hline & $\mathrm{Fe}^{3+}$ & $0,30(3)$ & $0,43(1)$ & & $2,6(2)$ & & \\
\hline & $\mathrm{DC}$ & 0,51 & $-0,04$ & & $28,1(2)$ & $0,39(2)$ & 36,95 \\
\hline & & & & & & & 39,33 \\
\hline & & & & & & & 42,49 \\
\hline & & & & & & & 44,40 \\
\hline & & & & & & & 47,0 \\
\hline
\end{tabular}

DC = distribuição de campo hiperfino, onde os valores são expressos como a média de grandeza. $\mathrm{Fe}^{3+}=$ paramagnético; $\mathrm{Mh}=$ maghemita; [Mf] e $\{\mathrm{Mf}\}$ - sítios tetraédrico e octaédrico da magnesioferrita, $A R=$ área relativa subespectral. 
numérico foi realizado com cinco subespectros: um dupleto, atribuído a $\mathrm{Fe}^{3+}$, pouco intenso, (super)paramagnético, e dois sextetos atribuíveis a $\mathrm{Fe}^{3+}$ na estrutura da magnesioferrita, um com deslocamento isomérico $\delta=0,45 \mathrm{~mm} \mathrm{~s}^{-1}$, em relação ao aFe, e $B_{h f}=51,60$ tesla, correspondente ao sítio de coordenação octaédrica e outro com $\delta=0,34 \mathrm{~mm} \mathrm{~s}^{-1}$ e $B_{h f}=52,98$ tesla, atribuído ao sítio de coordenação tetraédrica. Dois outros sextetos são atribuíveis, respectivamente, aos sítios de coordenação tetraédrico e octaédrico da maghemita, com deslocamentos isoméricos de $\delta=0,34$ e $0,32 \mathrm{~mm} \mathrm{~s}^{-1}$ e campos hiperfinos de $B_{h f}=49,48$ e 50,70 tesla, respectivamente.

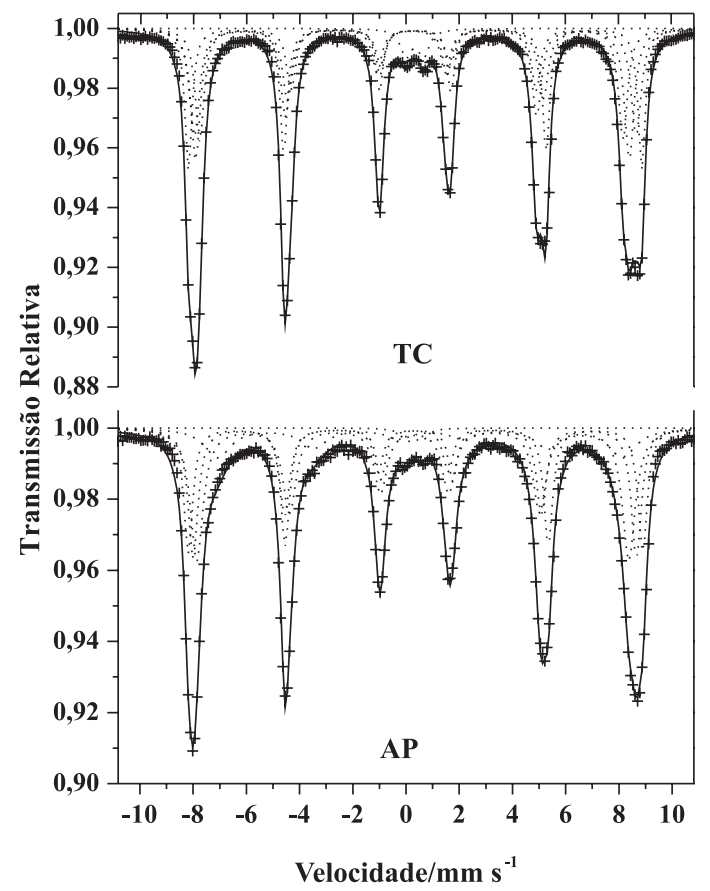

Figura 4. Espectros Mössbauer da fração magnética do tufito (TC) e do seu solo derivado (AP) obtidos a $110 \mathrm{~K}$

Tabela 4. Parâmetros Mössbauer dos espectros das amostras TC e AP, obtidos a $110 \mathrm{~K}$

\begin{tabular}{lccccc}
\hline Amostra & Sítio & $\delta / \mathrm{mm} \mathrm{s}^{-}$ & $\Delta, \varepsilon / \mathrm{mm} \mathrm{s}^{-1}$ & $B_{\mathrm{hf}} / \mathrm{T}$ & $A R / \%$ \\
\hline TC & $\mathrm{Fe}^{3+}$ & 0,43 & 0,66 & - & 3,2 \\
& $\{\mathrm{Mf}\}$ & 0,45 & $\sim 0$ & 51,6 & 23,0 \\
& {$[\mathrm{Mf}]$} & 0,34 & $0 *$ & 52,98 & 25,6 \\
& {$[\mathrm{Mh}]$} & 0,34 & $-0,07$ & 49,48 & 21,3 \\
& $\{\mathrm{Mh}\}$ & 0,32 & $-0,05$ & 50,70 & 27,0 \\
\multirow{4}{*}{} & $\mathrm{Fe}^{3+}$ & 0,41 & 0,55 & - & 1,2 \\
& $\mathrm{NC}$ & 0,83 & 0,40 & 47,20 & 14,6 \\
& {$[\mathrm{Mf}]$} & 0,40 & $0,06 *$ & 51,67 & 24,6 \\
& $\{\mathrm{Mf}\}$ & 0,44 & $-0,05$ & 52,95 & 25,2 \\
& {$[\mathrm{Mh}]$} & 0,38 & $-0,06$ & 50,35 & 34,4 \\
& $\{\mathrm{Mh}\}$ & 0,32 & $\sim 0$ & 50,8 & 11 \\
\hline
\end{tabular}

$\mathrm{Fe}^{3+}$ - superparamagnética. $\mathrm{NC}=$ fase não identificada, possível mistura de fases de Mf, Mh e Hm com pequeno tamanho cristalinos. [Mf] e $\{\mathrm{Mf}\}$ - sítios tetraédrico e octaédrico da magnesioferrita. $[\mathrm{Mh}]$ e $\{\mathrm{Mh}\}$ - sítios tetraédrico e octaédrico da maghemita, $A R=$ área relativa subespectral.
Considerando-se os parâmetros Mössbauer e a hipótese da ocorrência de magnesioferrita relativamente pura e estequiométrica nesse tufito, pode-se alocar o ferro e o magnésio nas proporções molares equivalentes à fórmula $\mathrm{Fe}_{2} \mathrm{MgO}_{4}$. Para a maghemita, considerando-se as possíveis substituições isomórficas, a composição será correspondente à formula $\mathrm{Fe}_{\mathrm{x}}^{3+} \mathrm{M}_{\mathrm{y}}^{\mathrm{c}+} \oplus_{\mathrm{z}} \mathrm{O}_{4}$, em que $\mathrm{M}^{\mathrm{c}+}$ representa os cátions substituintes isomórficos, $\oplus$ são vacâncias catiônicas e $\mathrm{x}+\mathrm{y}+\mathrm{z}=3$. Com base nas áreas espectrais Mössbauer e do difratograma de raios $\mathrm{X}$, distribuiu-se, igualmente, a proporção de ferro encontrada pelas análises químicas, entre as duas espécies. A alocação da fórmula química leva a: ${ }^{29}$

$\mathrm{Fe}_{2}^{3+} \mathrm{Mg}^{2+} \mathrm{O}_{4}$

$\mathrm{M}=200,00 \mathrm{~g} \mathrm{~mol}^{-1}$

Fórmula 1

$\mathrm{Fe}_{1,55}^{3+} \mathrm{Al}_{0,08}^{3+} \mathrm{Cr}_{0,01}^{3+} \mathrm{Mn}_{0,07}^{2+} \mathrm{Ni}_{0,07}^{2+} \mathrm{Ti}_{0,31}^{4+} \oplus_{0,13} \mathrm{O}_{4} \quad \mathrm{M}=195,07 \mathrm{~g} \mathrm{~mol}^{-1}$

Fórmula 2

A relação de áreas espectrais relativas encontrada para os sítios de coordenações tetraédrica $[T]([\mathrm{AR}])$ e octaédrica $\{\mathrm{M}\}$ ( $\{\mathrm{AR}\})$, para esta magnesioferrita, é de $\{\mathrm{Fe}\}]_{[\mathrm{Fe}]} \approx 1$. A fração livre de recuo, para o ferro em valência mista, no sítio cotaédrico (f $\{\mathrm{M}\}$ ) é $6 \%$ menor do que a do sítio tetraédrico (f[T]), para a da magnetita à temperatura do ambiente. ${ }^{26} \mathrm{Na}$ consideração de que a mesma diferença seja aplicável à magnesioferrita, a proporção molar de ocupação catiônica, nos dois sítios de coordenação será dada pela Equação 1

$\frac{\{\mathrm{Fe}\}}{[\mathrm{Fe}]}=\frac{\frac{\{\mathrm{AR}\}}{\mathrm{Fe}_{\{\mathrm{M}\}}}}{\frac{[\mathrm{AR}]}{\mathrm{Fe}_{[\mathrm{T}]}}}$

Assim, os dados correspondentes ao concentrado magnético do tufito (Tabela 1) inseridos na Equação 1 levam à razão $\{\mathrm{Fe}\}]_{[\mathrm{Fe}]} \approx 1,05$, para a magnesioferrita. A partir desta relação, a Fórmula 1 tem a seguinte distribuição iônica:

$\left[\mathrm{Mg}_{0,02}^{2+} \mathrm{Fe}_{0,98}^{3+}\right]\left\{\mathrm{Mg}_{0,98}^{2+} \mathrm{Fe}_{1,02}^{3+}\right\}_{\mathrm{O}_{4}}$

Fórmula 3

Considerando-se os demais cátions da Tabela 1, incluindo a parte residual de $\mathrm{Fe}^{3+}$ e de $\mathrm{Mg}^{2+}$, após a locação na magnesioferrita, como também os valores das áreas espectrais Mössbauer, e a relação \{Fe\}] ${ }_{[\mathrm{Fe}]} \approx 1,27$, é possível propor para a maghemita estudada a seguinte composição:

$\left[\mathrm{Al}_{\mathrm{x}}^{3+} \mathrm{Fe}_{0,68}^{3+} \mathrm{Mg}_{\mathrm{w}}^{2+} \mathrm{Mn}_{\mathrm{y}}^{2+} \mathrm{Ti}_{\mathrm{z}}^{4+} \mathrm{Cr}_{\mathrm{u}}^{3+} \mathrm{Ni}_{\mathrm{r}}^{2+}\right]$

$\left\{\mathrm{Al}_{0,08-\mathrm{x}}^{3+} \mathrm{Fe}_{0,87}^{3+} \mathrm{Mg}_{0,78-\mathrm{w}}^{2+} \mathrm{Mn}_{0,07-\mathrm{y}}^{2+} \mathrm{Ti}_{0,31-\mathrm{z}}^{4+} \mathrm{Cr}_{0,23-\mathrm{u}}^{3+} \mathrm{Ni}_{0,01-\mathrm{r}}^{2+} \otimes_{0,13}\right\} \mathrm{O}_{4}$

Fórmula 4

A análise qualitativa do espectro Mössbauer do concentrado magnético do solo derivado de tufito (amostra AP) tratado com DCB, a 110 K (Tabela 4 e Figura 4) revela também o ordenamento magnético de parte da fração superparamagnética, observável pela diminuição da intensidade do dupleto central e do aumento das áreas dos sítios cristalinos, como já observado anteriormente para a amostra TC. O ajuste do espectro Mössbauer da amostra a essa temperatura foi realizado com o emprego de cinco subespectros: um dupleto pouco intenso, atribuído a $\mathrm{Fe}^{3+}$, (super)paramagnético, dois sextetos atribuíveis a $\mathrm{Fe}^{3+}$ na estrutura 
da magnesioferrita, com deslocamento isomérico, em relação ao $\alpha \mathrm{Fe}$, $\delta=0,44 \mathrm{~mm} \mathrm{~s}^{-1}$ e $B_{h f}=52,95$ tesla, correspondente ao sítio de coordenação tetraédrica e outro, com $\delta=0,40 \mathrm{~mm} \mathrm{~s}^{-1}$ e $B_{h f}=51,67$ tesla, atribuído ao sítio de coordenação octaédrico do mineral. Dois outros sextetos, com campos hiperfinos, $B_{h f}=50,35$ e 50,80 tesla, são correspondentes à maghemita. Observa-se a presença de um quinto subespectro com $B_{h f}=47,20$ tesla, que pode ser atribuído a óxidos de ferro com partículas de tamanhos tão pequenos, a ponto de não atingirem o completo bloqueio da excitação magnética coletiva. ${ }^{28}$

O refinamento estrutural Rietveld da amostra AP é mostrado no difratograma de raios X numericamente ajustado da Figura 5. Para este refinamento, foram adotados dados dos modelos estruturais da magnesioferrita e da maghemita reportados na literatura. ${ }^{26}$ Para maior refinamento do difratograma, usou-se a função ThompsonCox-Hastings pseudo-Voigt * Axial divergence asymmetry, o que levou a uma melhor descrição das intensidades das fases individuais, indicadas pelo menor fator de $\chi^{2}=3,96$, para o concentrado magnético do solo. Os desvios dos fatores $R_{b}$ e $R_{f}$ de cada fase e os parâmetros finais do ajuste são mostrados nas Tabelas 5 e 6 .

Tabela 5. Dados obtidos a partir do refinamento estrutural Rietveld para a amostra AP

\begin{tabular}{lccccc}
\hline Mineral & $\begin{array}{c}\text { Proporção da fase } \\
\text { cristalográfica/\% }\end{array}$ & \multicolumn{4}{c}{ Fator } \\
$\mathrm{R}_{b}$ & $\mathrm{R}_{f}$ & $\mathrm{a} / \mathrm{nm}$ & $\mathrm{V}_{m} / \mathrm{cm}^{3}$ \\
\hline $\begin{array}{l}\text { Magnesio- } \\
\text { ferrita }\end{array}$ & $51,37(1)$ & 6,56 & 7,75 & $0,837563(2)$ & $587,56(2)$ \\
$\begin{array}{l}\text { Maghe- } \\
\text { mita }\end{array}$ & $48,63(1)$ & 5,77 & 8,01 & $0,835758(1)$ & $583,80(3)$ \\
\hline
\end{tabular}

Tabela 6. Coordenadas estruturais (x, y, z) e ocupação relativa (OR), obtidas para os modelos estruturais dos minerais presentes na amostra AP

\begin{tabular}{lcccc}
\hline Átomo & $x$ & $y$ & $z$ & OR \\
\hline \multicolumn{5}{c}{ Magnesioferrita } \\
{$[\mathrm{Fe}]$} & 0,12500 & 0,12500 & 0,12500 & 0,03799 \\
$\{\mathrm{Fe}\}$ & 0,50000 & 0,50000 & 0,50000 & 0,04767 \\
$\{\mathrm{Mg}\}$ & 0,50000 & 0,50000 & 0,50000 & 0,02361 \\
$\mathrm{O}$ & 0,26116 & 0,26116 & 0,26116 & 0,15563 \\
& & Maghemita & \\
{$[\mathrm{Fe}]$} & 0,12500 & 0,12500 & 0,12500 & 0,04251 \\
$\{\mathrm{Fe}\}$ & 0,50000 & 0,50000 & 0,50000 & 0,05942 \\
$\mathrm{O}$ & 0,26775 & 0,26775 & 0,26775 & 0,16667 \\
$\left\{\mathrm{Mn}^{2+}\right\}$ & 0,50000 & 0,50000 & 0,50000 & 0,00053 \\
$\left\{\mathrm{Ni}^{2+}\right\}$ & 0,50000 & 0,50000 & 0,50000 & 0,00064 \\
$\left\{\mathrm{Cr}^{2+}\right\}$ & 0,50000 & 0,50000 & 0,50000 & 0,00072 \\
$\left\{\mathrm{Ti}^{4+}\right\}$ & 0,50000 & 0,50000 & 0,50000 & 0,00517 \\
\hline
\end{tabular}

[ ] = sítio de coordenação tetraédrica; \{\} = sítio de coordenação octaédrica e $\mathrm{O}=$ oxigênio.

A análise quantitativa estimada da fração molar percentual magnesioferrita:maghemita para a amostra AP, a partir do refinamento Rietveld, é 51,3:48,7, que é próxima à relação obtida através da difração de raios X e das áreas Mössbauer.

Com base no refinamento estrutural, foi possível estabelecer a distribuição iônica na magnesioferrita. A fórmula estimada para a mesma foi

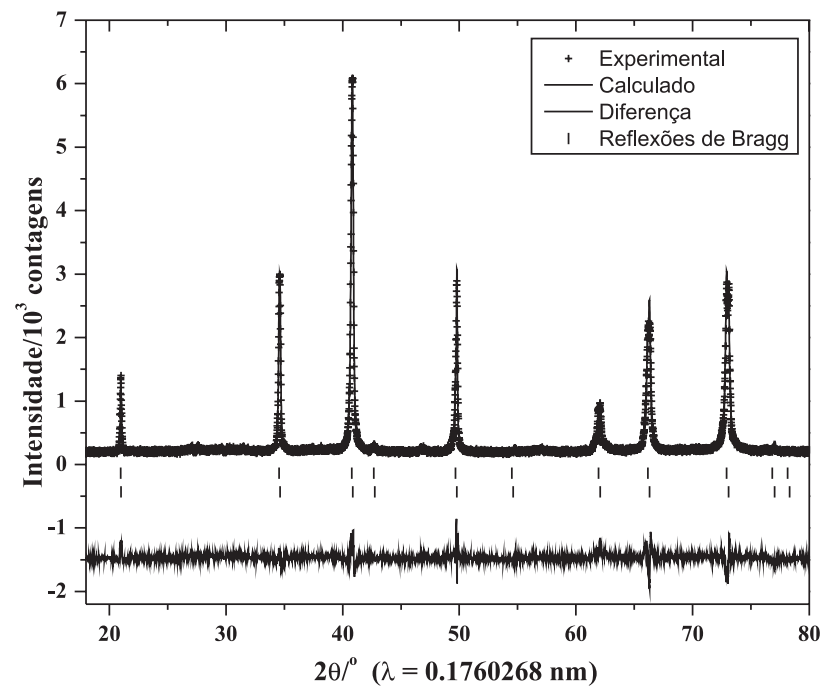

Figura 5. Refinamento estrutural Rietveld, obtido a partir do difratograma de raios $X$ da amostra AP (Figura 2)

$$
\left[\mathrm{Mg}_{0,03}^{2+} \mathrm{Fe}_{0,88}^{3+}\right]\left\{\mathrm{Fe}_{1,09}^{3+} \mathrm{Mg}_{1,0}^{2+} \otimes_{0,011}\right\} \mathrm{O}_{4} \quad\left(\mathrm{M}=199,55 \mathrm{~g} \mathrm{~mol}^{-1}\right)
$$

Fórmula 5

Este resultado é comparável ao obtido das análises químicas e da espectroscopia Mössbauer (Fórmula 3), exceto quanto a um pequeno desequilíbrio de cargas, provavelmente devido a uma deficiência de $\mathrm{Fe}^{3+}$, no sítio octaédrico, levando à relação $\mathrm{Fe}^{3+} / \mathrm{Mg}^{2+}=$ 1,98, e não a ideal, ${ }^{\mathrm{Fe}^{3+}} / \mathrm{Mg}^{2+}=2$, que seria esperada para uma estrutura estequiométrica. Como consequência, surgem vacâncias, aqui alocadas no sítio octaédrico. ${ }^{30}$

O parâmetro de rede da célula cúbica da magnesioferrita, presente na amostra AP, obtido por meio do refinamento Rietveld, é $\boldsymbol{a}=0,837563(2) \mathrm{nm}$. Silva et al. ${ }^{11}$ estudando o concentrado magnético de um tufito (material de origem do solo estudado), ineditamente caracterizaram magnesioferrita nesse mesmo material, obtendo através do refinamento Rietveld parâmetros de rede da ordem de $\boldsymbol{a}=0,83793(3) \mathrm{nm}$. A dimensão reportada de célula unitária cúbica para uma magnesioferrita sintética é $\boldsymbol{a}=0,8387(6)$ nm (ficha JCPDS (1980) número 36-0398). Observa-se uma diferença de $0,14 \%$, quando se compara a magnesioferrita sintética com aquela encontrada no solo estudado e de $0,05 \%$ quando se compara a magnesioferrita encontrada por Silva et al. ${ }^{11}$ à encontrada na amostra de solo, do presente estudo. Comparando-se os parâmetros de rede da magnesioferrita com os da maghemita (ficha JCPDS (1980) número 39-1346, dimensão característica da célula unitária cúbica, $\boldsymbol{a}=0,83512(6) \mathrm{nm}$ ), ambas estequiométricas, observa-se uma diferença de $0,43 \%$, quando se vai da magnesioferrita para a maghemita. Da magnesioferrita encontrada no solo para a maghemita estequiométrica há uma diferença de $0,28 \%$. Estes valores sugerem que durante os processos intempéricos, a magnesioferrita tende a uma maghemita com alto teor de substituição do ferro por magnésio $\left(\mathrm{MgFe}_{2} \mathrm{O}_{4} \rightarrow \gamma \mathrm{Mg}_{\mathrm{x}} \mathrm{Fe}_{2-\mathrm{x}} \mathrm{O}_{3}\right)$, mais genericamente nominável, uma $\mathrm{Mg}$-maghemita ${ }^{4-5}$. Silva et al. ${ }^{10}$ estudando um perfil de solo desenvolvido de tufito, observaram diminuição do tamanho de partículas e das áreas espectrais Mössbauer da magnesioferrita, quando se vai da base para o topo do perfil, com consequente aumento das áreas espectrais da maghemita e da magnetização de saturação do material, sugerindo, também, o mesmo caminho de transformação da magnesioferrita, como proposto neste trabalho. 


\section{CONCLUSÕES}

A análise mineralógica do concentrado magnético do tufito (TC) tratado com a mistura ditionito-citrato-bicarbonato (DCB), para o qual a magnetização de saturação medida foi $\sigma=14,0 \mathrm{~J} \mathrm{~T}^{-1} \mathrm{~kg}^{-1}$, mostra a ocorrência de maghemita rica em magnésio e de magnesioferrita, em proporções aproximadamente equimolares, que respondem pela magnetização observada do pedomaterial. A análise da amostra do solo derivado, AP $\left(\sigma=44,7 \mathrm{~J} \mathrm{~T}^{-1} \mathrm{~kg}^{-1}\right)$, tratada com $\mathrm{DCB}$, revelou a presença também de magnesioferrita e de maghemita. A partir dos parâmetros de rede cristalográfica cúbica, obtidos por refinamento estrutural Rietveld para a magnesioferrita $(\boldsymbol{a}=0,83756(3) \mathrm{nm})$ e para a maghemita $(\boldsymbol{a}=0,83575(2) \mathrm{nm})$, das amostras, propõe-se um modelo geral de transformação destes óxidos de ferro, em que a magnesioferrita tende a uma maghemita com alto teor de substituição do ferro por magnésio $\left(\mathrm{MgFe}_{2} \mathrm{O}_{4} \rightarrow \gamma \mathrm{Mg}_{\mathrm{x}} \mathrm{Fe}_{2-\mathrm{x}} \mathrm{O}_{3}\right)$. A magnesioferrita é, neste modelo, o mineral precursor pedogenético da maghemita, até a hematita, no pedossistema derivado de tufito: $\mathrm{MgFe}_{2} \mathrm{O}_{4} \rightarrow \gamma \mathrm{Mg}_{\mathrm{x}} \mathrm{Fe}_{2-\mathrm{x}} \mathrm{O}_{3} \rightarrow \alpha \mathrm{Fe}_{2} \mathrm{O}_{3}$.

\section{AGRADECIMENTOS}

À CAPES, ao UNIPAM, ao CNPq e à Fapemig pelo apoio financeiro. Ao Laboratório Nacional de Luz Síncroton (LNLS), em particular ao Dr. F. F. Ferreira por todo apoio na obtenção dos difratogramas de raios $\mathrm{X}$.

\section{REFERÊNCIAS}

1. Carmo, D. N.; Curi, N.; Resende, M.; R. Bras. Ci. Solo 1984, 8, 235.

2. Resende, M.; Allan, J.; Coey, J. M. D.; Annu. Rev. Earth Planet. Sci. 1986, 78, 322 .

3. Johannsen, A.; A descriptive petrography of the igneous rocks, The Univ. of Chicago Press: Chicago, 1969, vol. 1.

4. Fabris, J. D.; Coey, J. M. D.; Jesus Filho, M. F.; Santana, D. P.; Goulart, A. T.; Fontes, M. F.; Curi, N.; Hyperfine Interact. 1994, 91, 751.

5. Fabris, J. D.; Coey, J. M. D.; Mussel, W. N.; Am. Mineral. 1995, 80, 664.

6. Fabris, J. D.; Jesus Filho, M. F. de; Coey, J. M. D.; Mussel, W. N.; Goulart, A.T.; Hyperfine Interact. 1997a, 110, 23.

7. Fabris, J. D.; Mussel, W. N.; Coey; J. M. D.; de Jesus Filho, M. F.; Goulart, A. T.; Hyperfine Interact. 1997b, 110, 33.

8. Goulart, A. T.; de Jesus Filho, M. F.; Fabris, J. D.; Coey, J. M. D.; Phys. Chem. Miner. 1997, 25, 63.
9. Mussel, W. da N.; Fabris, J. D.; Coey, J. M. D.; Sans, L. M. A.; Lelis, M. F. F.; R. Bras. Ci. Solo 1999, 23, 779.

10. Silva, F. D.; Goulart, A. T.; Fabris, J. D.; Ker, J. C.; Couceiro, P. R. C.; Quim. Nova 2005, 26, 5 .

11. Silva, F. D.; Goulart, A. T.; Fabris, J. D.; Ker, J. C.; Couceiro, P. R. C.; R. Bras. Ci. Solo 2005, 29, 105.

12. http://talc.geo.umn.edu/orgs/irm/hg2m/hg2m_index.html, acessada em Março 2008.

13. EMBRAPA - Empresa Brasileira de Pesquisa Agropecuária; Sistema Brasileiro de Classificação de Solos, Embrapa Produção de Informação: Brasília, 1999.

14. Mehra, O. P.; Jackson, M. L.; Clays Clay Miner., National Conference Washington, 1958.

15. Neves, A. A.; Goulart, A. T.; Garotti, F. V.; Quim. Nova 1985, 8, 152.

16. Jeffery, P. G.; Hutchison, D.; Chemical methods of rock analysis, Pergamons: London, 1981.

17. Coey, J. M. D.; Cugat, O.; Mccauley, J.; Fabris, J. D.; Revista de Física Aplicada e Instrumentação 1992, 7, 25.

18. Young, R. A.; The Rietveld Method; University Press: Oxford,1995.

19. Carvajal, R. J.; Sattellite Meeting on Powder Diffraction, Toulouse, 1990.

20. Caglioti, G.; Paoletti, A.; Ricci, F. P.; Nucl. Instrum. Methods Phys. Res. 1958, 16, 223.

21. Brand, R. A.; Laboratórium för Argewandte Physik, Universität Duisburg: Duisburg, 1990.

22. JCPDS - Joint Committee on Powder Diffraction Standards; Mineral Powder Diffration Files Data Book, Swarthmore: Pennsylvania, 1980.

23. Murad, E.; Johnston, J. H.; Mössbauer Spectroscopy Applied to Inorganic Chemistry, Plenum: New York, 1987, vol. 2.

24. Cornell, R. M.; Schwertmann, U.; The iron oxides, Weinheim: VHC, 1996.

25. Hamdeh, H. H.; Xia, Z.; Foehrweiser, R.; McCormick, B. J.; Willey, R. J.; Busca, G.; J. Appl. Phys. 1994, 76, 1135.

26. Sawatzky, G. A.; Woude, F.; Morrish, A. H.; Phys. Rev. 1969, 183, 747.

27. De Grave, E.; Bowen, L. H.; Weed, S. D.; J. Magn. Magn. Mater. 1982, $27,98$.

28. Morup S.; J. Magn. Magn. Mater. 1983, 39, 45.

29. http://moss.qui.ufmg.br/ Moss, acessada em Agosto 2008.

30. O’Neill, H. St. C.; Annersten, H.; Virgo, D.; Am. Mineral. 1992, 77, 725. 